\title{
COPING STRATEGIES OF MOTHERS WITH ASD CHILDREN
}

1. Professor of Bio Chemistry, Federal Medical \& Dental College, Islamabad.

2. Dean, Allied Health Sciences, UOL, Lahore

3. Assistant Professor,

Shifa Eye Trust Hospital,

Islamabad.

4. PIQC, Lahore

5. PIQC, Lahore.

Correspondence Address:

Dr. Hamid Mahmood

Professor of Bio Chemistry,

Federal Medical \& Dental College, Islamabad.

drhamidmahmood373@gmail.com

Article received on:

02/09/2015

Accepted for publication:

03/11/2015

Received after proof reading:

$13 / 11 / 2015$

\section{Dr. Hamid Mahmood', Prof. Dr. Munir Saleemi², Dr. Hashim Riaz ${ }^{3}$, Yasir Hassan ${ }^{4}$, Farzana Khan ${ }^{5}$}

ABSTRACT... Introduction: Autism spectrum disorder (ASD) is a group of developmental disabilities that can cause significant social, communication and behavioral challenges". American Psychiatric Association's defined ASD as "a single disorder that includes disorders that were previously considered separate - autism, Asperger's syndrome, childhood disintegrative disorder and pervasive developmental disorder not otherwise specified". Objectives: To exploration of the coping strategies of mother who are the primary care giver of the family and children with ASD. This study correspondingly emphasized on the policy makers to bring this subject to the agenda for the training (Coping) and support (Insurance) of mothers and ASD children for special education needs (SEN) by the government. Study Design: Qualitative study. Methods: This research explored mothers coping practices with ASD children in Rawalpindi by means of qualitative study design phenomenology. Theoretical framework used were, life course perspective and social constructivism which will facilitate the complete process. Interview guide is made on the format suggested by Steinar Kvale. Data analysis will be done by two analytical techniques, grounded theory analysis. Results: The results of this study were categories which were generated were divided into two main parts positive/ healthy and negative/impaired coping strategies they are as follow:- Positive coping strategies. a) Integration b) Networking c) Religion. And the Negative coping strategies a) Avoidance b) Ignorance c) Isolation. Conclusion: Overall, findings indicated that mothers sorrow and grief associated with ASD is common in every mother. Mothers coped by educating themselves. Findings indicated feelings of shock, grief and acceptance were the part of mother's responses, on the discovery of their child's autism diagnosis. Findings indicated daily routines were not affected but the ASD child.

Key words: $\quad$ Autism spectrum disorder (ASD)

Article Citation: Mahmood H, Saleemi M, Riaz H, Hassan Y, Khan F. Coping strategies of mothers with ASD children. Professional Med J 2015;22(11):1454-1463. DOI: 10.17957/TPMJ/15.3078

\section{INTRODUCTION}

"Autism spectrum disorder (ASD) is a group of developmental disabilities that can cause significant social, communication and behavioral challenges". Presently ASD is defined by the American Psychiatric Association's Diagnosis and Statistical Manual of Mental Disorders (DSM-5) as "a single disorder that includes disorders that were previously considered separate - autism, Asperger's syndrome, childhood disintegrative disorder and pervasive developmental disorder not otherwise specified". The term "spectrum" in autism spectrum disorder refers to the wide range of symptoms and severity. Although the term "Asperger's syndrome" is no longer in the DSM, some people still use the term, which is generally thought to be at the mild end of autism spectrum disorder.

\section{Characteristics of ASD}

ASD varies greatly in symptoms ranging from mild to extreme abnormalities like language for communication, social interaction and repeated or restricted behavior. The signs and symptoms can be explained in three broader areas. First social skills, uncommon or unsuitable body language, movements and facial expressions. Lack social interaction, struggle in understanding other people's feelings if they are not intense, and nonverbal cues, does not like to be touched and trouble or failure to make friends with children of same age. Second speech and language or 
doesn't speak at all, Speaking in an strange tone of voice, or with an unusual rhythm or pitch, Repetition of words or phrases without intension, suffer in starting a conversation and when started talks continuously, it's difficult for those children to express their needs, simple statements or questions are hard to understand. Takes very seriously what is said, missing humor, irony, and sarcasm. Third, restricted behavior and play. Concern with a specific topic of interest for example numbers or symbols (maps, license plates, sports statistics), a strong need for similar orders and routines (e.g. lines up toys, follows a rigid schedule). If the routine or environment is changed gets distressed. Awkwardness, strange posture, or unusual ways of moving and fascinated by spinning objects, moving pieces, or parts of toys. Center for Autism Research \& Treatment). The severity in signs and symptoms of ASD patient tends to fluctuate throughout the developmental age. ${ }^{1}$

\section{Onset of ASD}

The very first warning sign of ASD symptoms is in early first year of life which can comprise any of the symptoms mentioned above. This situation is known as "Regression". In regression loss of previously acquired skills occur generally prior to 24 months of age.

\section{Diagnosis}

Diagnosis of ASD is not an easy task owing to complication of symptoms; moreover there are no objective laboratory evidence that can confirm the diagnosis. This method of assessment can be used in a range of ages normally from 15 months to 40 years.

\section{Prognosis of ASD patients}

The future of ASD child depends on the severity of symptoms. The more intensive intervention incorporated to control the symptoms the better prognosis will result. According to some experts, some factors such as I.Q. level above 50 , ability to speak before age 6 and having a skill can be useful in better prognosis.

Interventions and Strategies for parents with ASD child
Therapies usually focus on educational and behavioral interventions for improvement in behavior. Like applied behavior analysis (ABA) which is used as a technique to encourage positive behavior and discourage negative behavior to improve variety of skills. ABA is further expanded in four parts first, discrete trial training (DTT) this is a teaching technique that utilizes a series of trails to teach the desired behavior or response. Second, early intensive behavioral intervention (EIBI) this is used for younger children less than age 3 or 5 . Third, pivotal response training (PRT), in which intentions are to increase a child's motivation to learn, and monitor his own behavior. Fourth, verbal behavior intervention (VBI) used to teach verbal skills to child. Other interventions used in ASD are occupational therapy, sensory integration therapy, speech therapy, music therapy, picture exchange communication system (PECS).

In Pakistan all the special school which are now licensed to train and educated the special children use speech therapy, occupational therapy, behavioral therapy, vocational therapy, $A B A$, and PECS. These interventions are provided according to the resources available. As Pakistan is under developed country, however, presently all the schools and hospitals allot degree holders and experienced people for the children and parents desired outcomes. Continuous and consistent therapy along with aid of expert professionals for ASD children is needed parents report in a study by Siklos \& Kerns, "After the child is diagnosed with ASD $87.3 \%$ of families utilized speech therapy which is the most common therapy followed by $67.5 \%$ occupational therapy, $45.6 \%$ utilized behavioral management programs while $42.7 \%$ applied learning strategies and study skills assistance". ${ }^{2}$

\section{LITERATURE REVIEW}

Global Burden of ASD

(Centers for Disease Control and Prevention 2014)

ASD is estimated to affect 1 in 68 children in many communities in USA. ASD is reported to occur in all racial, ethnic, and socioeconomic groups. ASD is almost 5 times more common among boys (1 in 42) than among girls (1 in 189) according to 
estimates from CDC's Autism and Developmental Disabilities Monitoring (ADDM) Network. Studies in Asia, Europe, and North America have identified individuals with ASD with an average prevalence of about $1 \%$. A study in South Korea reported a prevalence of $2.6 \%$. About 1 in 6 children in the United States had a developmental disability in 2006-2008, ranging from mild disabilities such as speech and language impairments to serious developmental disabilities, such as intellectual disabilities, cerebral palsy, and autism. The prevalence of autism in Lahore, Pakistan is $6.31 \%$ but this research is not authentic in its generalization. ${ }^{3}$

\section{Parenteral Stress}

Parents having ASD children will go through changes in family dynamics, routines and activities had to be altered according to ASD child. Each family cope in different manner, just as the symptoms fluctuates, so does each family experience.

ASD Diagnosis is a life changing experience, which can change their perspective of parents to life. It can give positive result giving strength based perspective. ASD can result in developing empathetic and compassionate element in parents and siblings to view ASD children to be different. When the parents of child with ASD and with other DD like Down syndrome etc. are compared stress levels are high in ASD parents. The reasons which increases the stress of parents with ASD children can be greater time consumption in care and therapy like speech, language and occupational therapy which results in negligence of other important matters. Anxiety is another mental health concern of parents of ASD child and symptoms are reported in and depression. Parents of children with ASD experience more intense child behavior problems. The divorce rate in couples with ASD children are high compared to the population parenting children with other disabilities.

\section{ASD and Siblings}

Association of ASD child and siblings cannot be denied, there are numerous stressors which can contribute to siblings stress. Stressor like embarrassment, jealousy, frustration, grief of parents related to ASD and future concerns. Sibling complaint of negative outcome like embarrassment among peers due to their behavior when one have ASD sibling. When the sibling of ASD and metal retarded were compared, siblings of ASD reported more problems regarding disturbance or breaking things as compared to other.

Frustration leading to aggression is seen among the siblings of Autistic child. Siblings ranging between 8-15 years reported aggression when trying to intermingle with ASD sibling. Siblings reported major concern regarding the future of their autistic sibling. ${ }^{4}$

\section{Coping Strategies}

The phrase coping implies the process of managing stressful circumstances. The parents and family starts employing coping strategies soon after the diagnosis of the child with ASD. Coping strategies like problem-focused and emotion-focused coping strategies 7 are used by parents. Problem-focused strategy is mostly adopted by fathers or males which involve cognitive and behavioral strategies to change the situation, these coping efforts are based on the stressful situation itself. Emotion-focused strategies are adopted mostly by mothers or females which involve social support, expression of emotions and communication of feeling as a reaction to stressful situations which is the key to these coping strategies.

\section{METHODOLOGY}

\section{Study design}

This was a qualitative research the research design selected is phenomenology, "which is the study of human phenomena, of things or events in everyday world. The participant (Mothers) in this research study of coping strategies of mothers with ASD child shared their experiences.

\section{Theoretical framework}

The theoretical frame works used include social constructivism to explicitly explain the contextual position of the participant. Social constructivism is a term which refers to how the people perceive certain things like ASD and life with ASD. The life course perspective (LCP) is also used as a theo- 
retical framework. LCP will gave a path to understand the change in mother's life after ASD diagnosis. The five stages of LCP gives chronological sequence of participant's lives before and after ASD diagnosis.

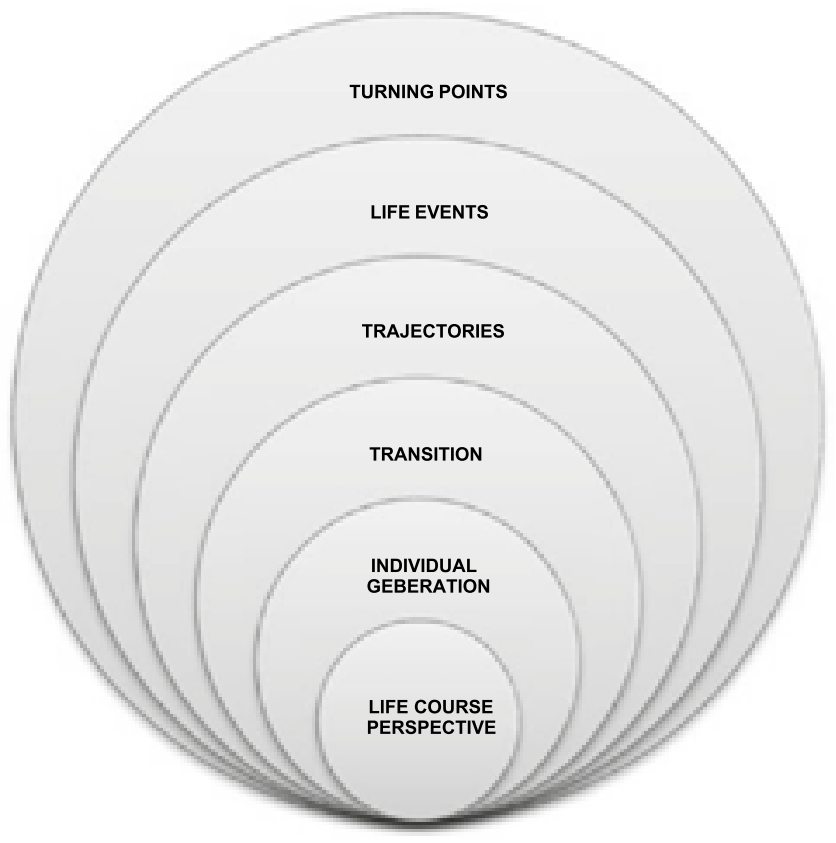

Social constructivism Social constructivism

\section{Study Site}

This research was carried out in Rawalpindi (research setting) Punjab. The target population (mothers) selected from special schools which includes three special schools of Rawalpindi city.

\section{Sample Size and Sampling Technique}

Phenomenology requires the participants should range from 5-25 individuals who have experienced the phenomenon. Sampling was purposive sampling and snow ball sampling, 10 mothers of ASD diagnosed children were included in which three were selected via snowball, in the research as the saturation level was attained where no new finding and information was acknowledged. Thus the interviews were stopped, but the situation would not have been same if the mother who did not wanted to participate in the research were included. More variation in participants would have given the researcher more information and finding.

\section{Data Collection}

Data collection was done in two stages pilot and post pilot, the pilot stage in which one mother was selected and in-depth interviews was conducted following transcription, coding and then analysis for deciding the appropriateness of the data collection technique. Some modifications were done on the interview guide with addition and omission of few questions. The interview guide comprised of open ended questions following the format suggested by Steinar Kvale. This was to fulfil the intention of rich data collection. The interviews were audiotaped. The interview was conducted in the national language of Pakistan "Urdu" and thus it was transcribed to roman English. The purpose of this step was to save the data from loss of meaning if translated to English language. The data collection and transcription was done concurrently to ensure rigor in the data. Interview duration range varied with each mother ranging from 50- 60 minutes. The interviews were in-depth and were done according to the convenience of the mothers, 2 interviews in the school, 5 interviews in the home of the participants and rest 3 were interviewed on phone call. After data collection 5 interviews with same participants were repeated in cases where the recording was inaudible and difficult to code.

\section{Methods of Analysis}

After selection of the topic for Research pilot study was done and presented in the presence of all the faculty of Al Shifa Trust Eye Hospital. Thus process termed as peer debriefing was done to ensure the feasibility and appropriateness of the research. The data collection was done longside analysis, which resulted in the amendment in interview guide and sampling in the very beginning of the study period. The data collected from indepth interviews were transcribed and line wise coded. Analysis (coding) was done by open code software, it does the analysis line wise for the verbatim. Those coding were compiled and categories were generated to grasp the essence of their lives. Constant comparative method of data analysis was used. The same steps were repeated with further interviews including additional questions to explore the phenomena in detail. Grounded theory analysis was done to extract out the coping strategies adopted by different mothers. 


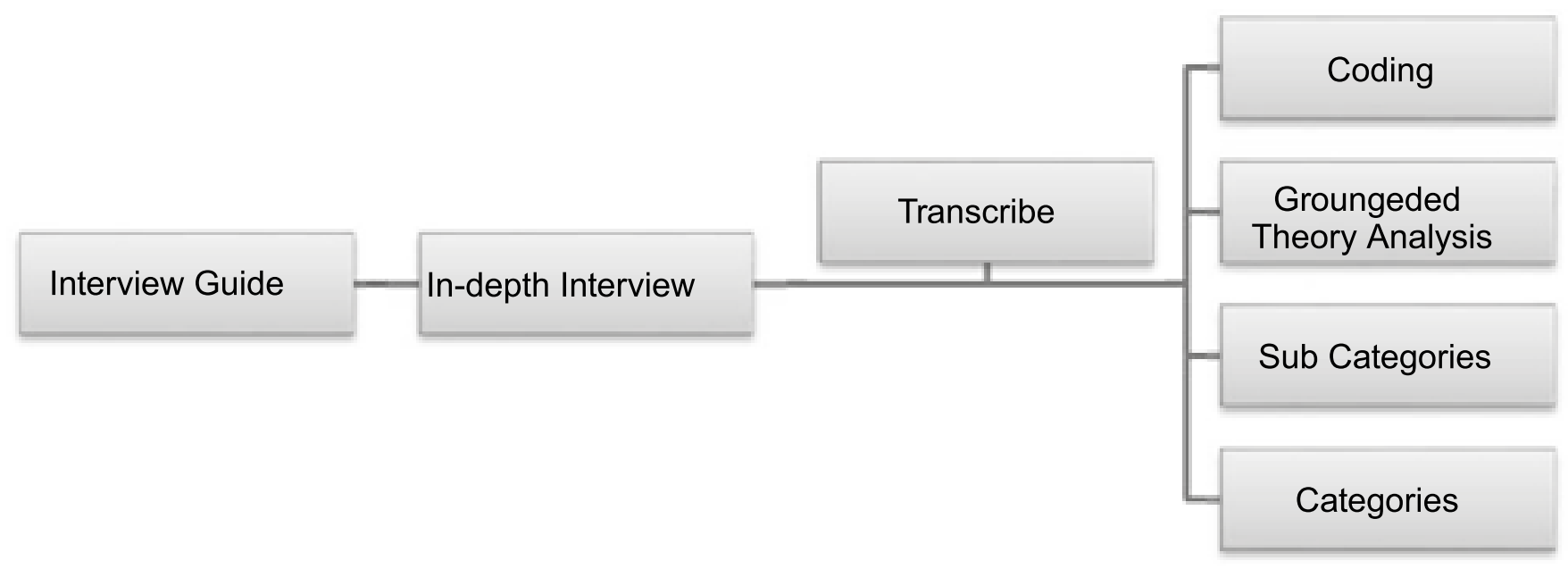

\section{Trustworthiness}

\section{Credibility}

Rich data was collected, which means enough data was collected until the saturation level was achieved. 10 participants were interviewed (indepth) to ensure that rich data is collected and maximum variation in perceptions of mothers regarding coping strategies was obtained.

Respondent validation or cross referencing, this involves reading the draft or asking extra time from participant to reconfirm the transcription to make sure that the researcher understood the meaning of the story told by the interviewee and to ensure that misinterpretation does not exist in the results.

Triangulation was used for the validity testing of the qualitative research. This method involves the analysis by different perspectives; this included my supervisor, colleagues and family.

Negative cases or deviant cases were filtered out elements, themes or concepts which do not match the research question. This analysis helps to keep the study purely to the related themes and contents of the research question or study.

\section{Transferability}

Transferability means research finding achieved should be as such that it can be transferred to any situation. It is vital that the interpretation of the phenomenon under inquiry should be explicit which allows the readers to understand, thus they can compare the cases with their own experiences.

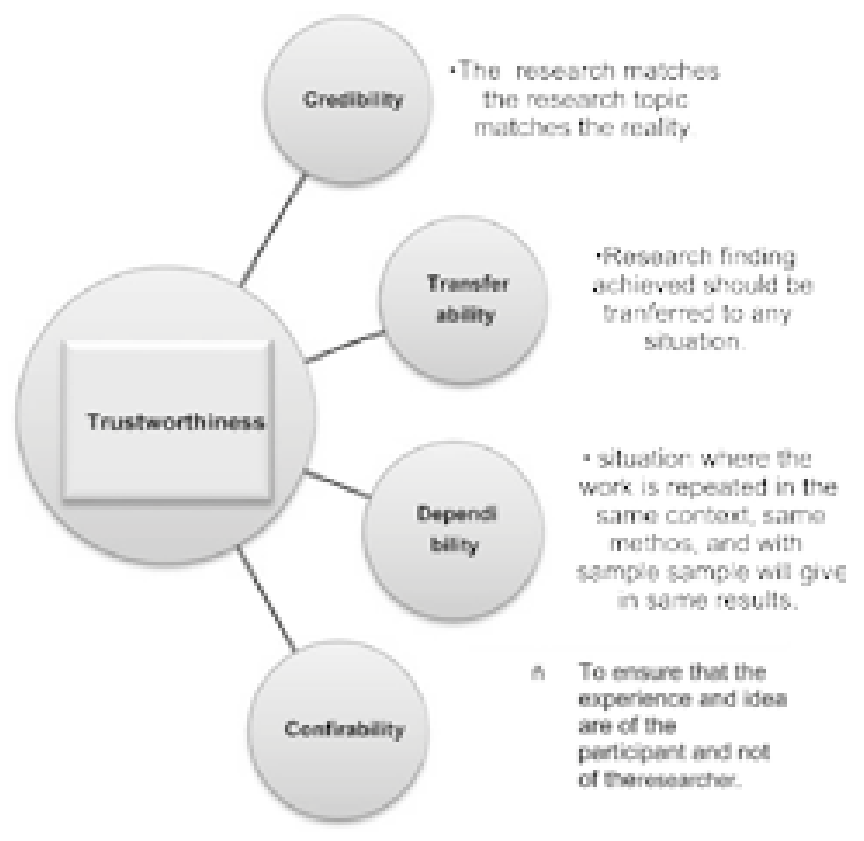

\section{Dependability}

Dependability refers to the situation where the work is repeated in the same context, with the same method, and with the same sample, similar results will be obtained. ${ }^{7}$

\section{Confirmability}

Confirmability is to ensure that the experience and idea used in the research are of participants, rather than the inclinations of researcher. Triangulation is used in supporting confrmability to reduce the effect of investigator bias. 


\section{Study Duration}

The study duration was from April to July. Starting from April which was devoted to data collection, transcription and coding through in-depth interviews. May and June was for analysis of the data collected.

\section{Ethical Consideration}

All the participants in the study were ensured for the protection and confidentiality of this research. All the consent form signed by the mothers are also protected.

\section{RESULTS}

This qualitative research comprised of 10 indepth interviews of mothers with ASD children. These interviews were conducted to explore the lives of mother after ASD diagnosis. Among the 10 mothers 4 mothers were employed and 6 were housewives, this factor influenced the coping strategies significantly. The mothers who were educated and had other responsibilities apart from taking care of ASD child were noticeably less stressful. Education and knowledge of ASD had very positive impact on the mothers and took this as a challenge, while the mothers who were in home were more superstitious which lead them to stress weak coping strategies.

In the interviews main categories which were generated were divided into two main parts positive/ healthy and negative/impaired coping strategies they are as follow:-

Positive coping strategies

a) Integration b) Networking c) Religion

Negative coping strategies

a) Avoidance b) Ignorance c) Isolation

\section{Integration}

Integration here refers to the internalization of ASD child into the normal life of parents and siblings. The change in the attitudes of parents towards the ASD child, that he is unique but part of our family.

\section{Networking}

The second positive coping strategy was networking; this term includes socializing and edu- cation which mothers adopted to cope with ASD. THE MOTHERS educated themselves and other parents with ASD children about the disorder and positively dealing with it.

\section{Religion}

The third positive coping strategy by the mothers were religion and majority of mothers if not strongly religious but have adopted this method in coping with the ASD situation.

\section{Avoidance}

Some Mothers in this study adopted a strategy which not completely but may be partially kept them out of the pain of ASD. They avoided the talk, thought or almost anything which made them think about ASD. They took ASD as part of the life but in healthier way.

\section{Ignorance}

This coping strategy was unhealthy for the mothers to adopt not only for them but the ASD child. The term ignorant was used because the mothers adopted very immature coping strategy to cope with $A S D$, which made them ignorant of the main priorities.

\section{Isolation}

This category emerged from the experience of the mothers who were socially restricted due to ASD child. The isolation was not only for mothers but the whole family including ASD child. The mother used the method of detachment from the ASD child.

\section{DISCUSSION}

In this research, the coping strategies of mothers were examined. What are the experiences of mothers with a child diagnosed with ASD? We used the study design phenomenology. This study indicates that mothers are the primary care giver and spend maximum time with ASD child as compared to other member of the family.

According to one study, when parents heard of the child' ASD diagnosis the feeling of the parents were " $52 \%$ felt relieved, $43 \%$ felt grief and loss, $29 \%$ felt shock or surprise, and $10 \%$ felt self- 


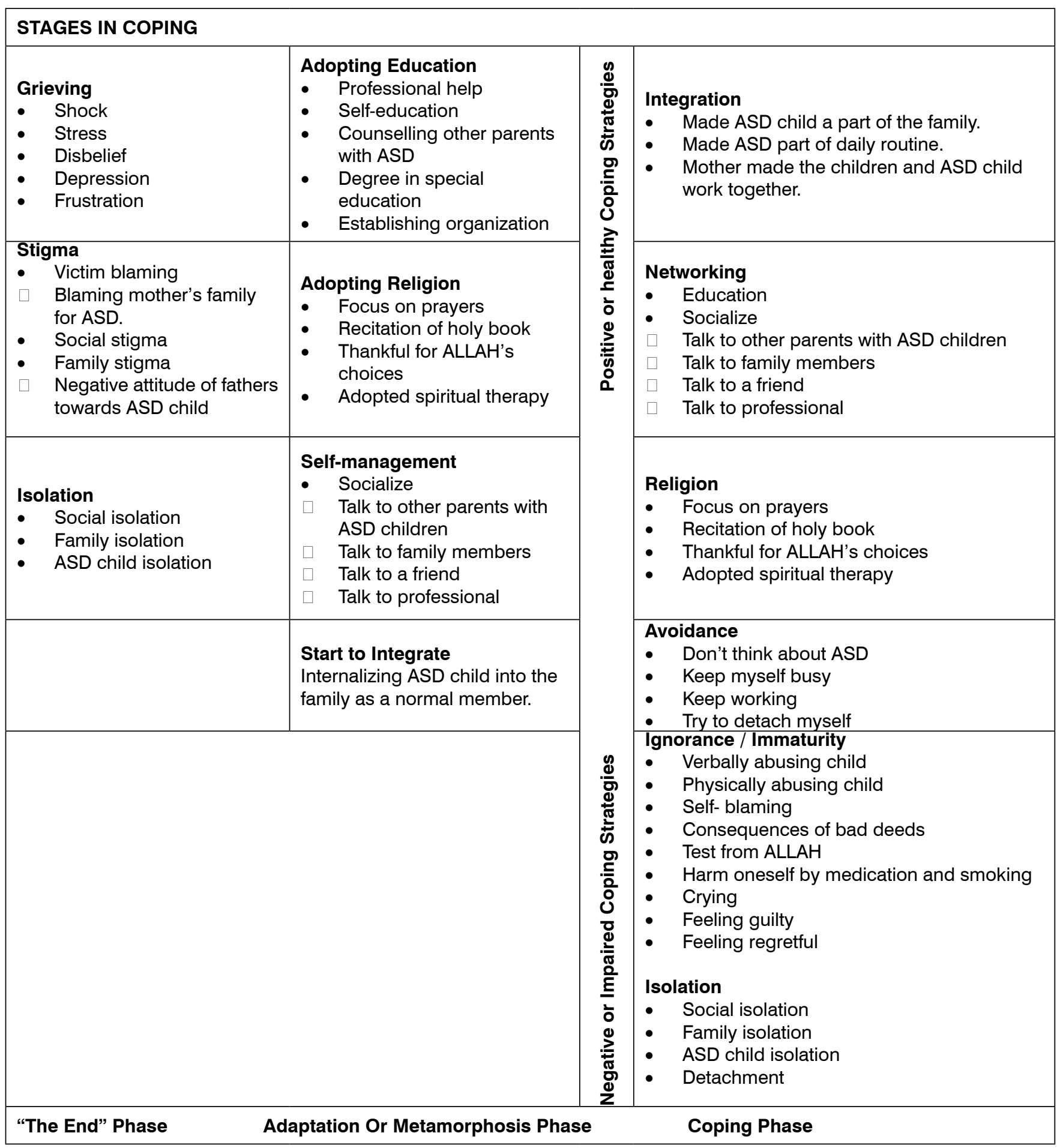

blame" this research cannot express the results in percentage but grief, shock and self-blaming was very prominent findings. Mother went through shock who never heard of autism, and as soon as they educated themselves about it they were grieving about it as it is lifelong disability. Mothers also experienced victim blaming.

ASD can result in developing empathetic and compassionate element in parents and siblings to view ASD children to be different. This research indicates that the empathetic and compassionate 
element was seen in almost every mothers when it was diagnosed but with acceptance and positive coping this is the element reported by the mothers. In one research mother reported that she feels she gave ASD child more care and attention as compared to other children.

The stress of parents with ASD children can be greater time consumption in care and therapy like speech, language and occupational therapy which results in negligence of other important matters. Very positive finding in this study which can relate to this research is the management of the daily routine with ASD child all the therapy, school timing, lunch etc. was managed which did not finish but considerably reduced the stress levels. Sibling complaint of negative outcome like embarrassment among peers due to their behavior when one have ASD sibling.

Coping strategies like problem-focused and emotion-focused coping strategies are used by parents. In this research the mix results were discovered those mothers who adopted the problem focused coping strategies were educated or have educated themselves after the diagnosis while those adopting the emotion focused coping strategy were mostly young mothers with education level low. Thus the finding here is education and awareness about ASD have made the mothers positively capable to manage the situation. The challenges faced by the parents especially mothers who are contributing more time and energy in the care of the child often have to play multiple roles like advocate, educator and caregiver. The mother or women as the gender responsibility is more likely carry the burden of the disable family member. This qualitative research agree to all the research, the mothers here are the primary care giver of the family and especially any disable child if present in the family. The mothers definitely plays multiple roles in this research they were advocators all the educated mothers when adopted any coping strategies which had positive results preached it to other mothers too, educator and care giver is the role which is evident from the coping strategies mentioned already.
The Women are more prone to be influenced by the stressful events occurring in the life as compared to men. The mothers in this research reported that the stress and the responsibility is more on the mothers. Fathers are usually responsible of the financial aid for the schooling, food and treatment of all the children. The fathers play role in motivating the ASD child towards sports and physical activities reported by mothers. "His father takes him for swimming"

\section{Future Research}

The research focuses on mothers coping strategies it can produce more topics which may include the father and siblings. This research focused on the families who were sending the ASD children to schools means they were affording families. Those families who are not affording or low income families should be studied. This would include families who have ASD children but don't afford to train their child. Policy can be very interesting topic any policy which can be designed for those children and implemented by the government. Group homes or organization which can support those children after the death of their parents will be good initiative. This research was done in a Rawalpindi; Pakistan is a large under developed country with numerous beliefs and norms many qualitative topics can be emerged based on cultural belief and Autism. Specifically, how diverse cultures experience the autism diagnosis and their cultural beliefs regarding ASD.

\section{Strengths}

Strength of these findings is the empowerment and knowledge provided current and future mothers. Personally I have learnt a lot about ASD and the mothers who were so strong that they can become motivation for the future mothers for coping and survival. Findings provide current and future families who are living with a child or sibling diagnosed with autism information on others personal encounters with this diagnosis. Findings also allow current and future families to see the thoughts, feelings and perspectives of those who have lived or are currently living with a child or sibling who has autism. 


\section{Limitations}

One limitation in these study was that majority of the mother whom I approached were not interested in participation. This did not allow the research to be versatile in terms of finding. The results may have diverse if there were perceptions from a larger sample size. These findings are principally viewed through the female lens. The number of male children in the research was greater with only 2 female ASD children.

Another limitation is the ethnic backgrounds of the mothers. Only one mother was Pashtun and all the rest were Punjabi. Ethnicity is a critical aspect which deals with the way mother cope with certain situations, the knowledge and cultural belief.

\section{CONCLUSIONS AND WAY FORWARD}

Overall, findings divided into two main parts the positive or healthy and negative or impaired coping strategies. Findings indicated feelings of shock, grief and acceptance were the part of mother's responses, on the discovery of their child's autism diagnosis. Mothers adopted a very brilliant and positive method to cope especially those who thoroughly knew the symptoms of ASD, they used the method of making the ASD child normal part of the family and routine. This method was given the name of integration. Mothers also coped by educating themselves most interesting finding was that those mothers who were aware and have prepared themselves through education adopted positive coping strategies. Diverting themselves toward religion was another finding. Avoidance was to escape the stress associated with ASD, by adopting the denial element: avoid thinking, talking etc. and just keeping oneself busy in work. Every mother reported ASD is a social stigma and victim blaming which is the main stressor and make the mothers more depressed. This stressor made them ignorant influenced them towards negative coping strategy.
Verbally and physical Abusing child, self-blaming, crying, consequences of bad deeds etc. thus this resulted in two main categories of the research study for coping strategies of mothers.

Copyright (C) 03 Nov, 2015.

\section{REFERENCES}

1. L. Zwaigenbaum, A. Thurm, W. Stone, G. Baranek, S. Bryson, J. Iverson, A. Kau, A. Klin, C. Lord, R. Landa, S. Rogers, M. Sigman. (March 2007) Studying the Emergence of Autism Spectrum Disorders in High risk Infants: Methodological and Practical Issues, Journal of Autism and Developmental Disorders (Volume 37, Issue 3, pp 466-480).

2. J. R. MacFarlane, T. Kanaya (2009) What Does it Mean to be Autistic? Inter-state Variation in Special Education Criteria for Autism Services, Journal of Child and Family Studies (Volume 18, Issue 6, pp 662-669).

3. Suhail K and Zafar F. Prevalence of autism in special education schools of Lahore. Pakistan journal of psychology research. 2008 vol. no. 3-4, 23 45-65p.

4. Shattuck, M. M. Seltzer, J. S. Greenberg, G. I. Orsmond, D. Bolt, S. Kring, J. Lounds, C. Lord (2007) Change in Autism Symptoms and Maladaptive Behaviors in Adolescents and Adults with an Autism Spectrum Disorder Journal of Autism.

5. S. Siklos, K. A. Kerns (2006) Assessing Need for Social Support in Parents of Children with Autism and Down Syndrome, Journal of Autism and Developmental Disorders (Volume 36, Issue 7, pp 921-933).

6. G. M. Griffith, R. P. Hastings, S. Nash, C. Hill (May 2010) Using Matched Groups to Explore Child Behavior Problems and Maternal Well-Being in Children with Down syndrome and Autism. Journal of Autism and Developmental Disorders (Volume 40, Issue 5, pp 610619).

7. S. Siklos, K. A. Kerns (2006) Assessing Need for Social Support in Parents of Children with Autism and Down Syndrome, Journal of Autism and Developmental Disorders (Volume 36, Issue 7, pp 921-933).

8. S.T. Sigmon, A. L Stanton, \& C. R Snyder. (1995). Gender differences in coping: A further test of socialization and role constraint theories. Sex Roles, (Volume 33, issue 9, pp 565-587). 


\section{5 \\ "The greatest enemy of knowledge is not ignorance. It is the illusion of knowledge."}

\section{Stephen Hawking}

\section{AUTHORSHIP AND CONTRIBUTION DECLARATION}

\begin{tabular}{|c|l|l|l|}
\hline Sr. \# & \multicolumn{1}{|c|}{ Author-s Full Name } & \multicolumn{1}{|c|}{$\begin{array}{c}\text { Contribution to the paper } \\
\text { Researcher, Data Collection }\end{array}$} \\
\hline 2 & Hamed Mahmood & Munir Saleemi & Research supervision \\
\hline 3 & Hashim Riaz & Data analysis \\
4 & Mr. Yasir Hassaan & Statical supervisor \\
5 & Farzana Khan & Data analysis
\end{tabular}

